\title{
Sarcoid Tattoo Granuloma and Ocular Sarcoidosis: The Great Masquerader A Maharaj ${ }^{1}$, J Cook $^{2}$, SA Lalchan ${ }^{3}$, M Suite ${ }^{4}$, S Barrow ${ }^{5}$
}

\author{
Affiliations: \\ ${ }^{1}$ Mt Hope Hospital, Champs Fleurs, Trinidad and Tobago \\ ${ }^{2}$ SUNY Downstate Medical Centre, Brooklyn, New York 11203. \\ ${ }^{3}$ LILY-The Eye Specialist Ltd, Chaguanas, Trinidad and Tobago. \\ ${ }^{4} 74$ Dundonald St, Port-of-Spain, Trinidad and Tobago. \\ ${ }^{5}$ Port-of-Spain General Hospital, Trinidad and Tobago.
}

\author{
Correspondence: \\ Ms S Lalchan \\ \#8 Endeavour Road \\ Chaguanas \\ Trinidad and Tobago \\ West Indies \\ E-mail: mslalchan@gmail.com
}

Short title: Bilateral Uveitis and Sarcoid Tattoo Granulomas 


\section{ABSTRACT}

Sarcoidosis is a multisystem disease with both sight and life-threatening sequelae. It can be a diagnostic challenge as there is no definitive test. The IWOS has recommended guidelines to improve diagnostic certainty to improve early detection. The authors present a case of bilateral panuveitis and biopsy proven sarcoid tattoo granuloma. The roles of a multi-disciplinary team and early introduction of steroid-sparing agents are highlighted.

Keywords: sarcoidosis, uveitis, sarcoid tattoo granuloma.

\section{INTRODUCTION}

Sarcoidosis is a multi-systemic inflammatory disorder of unknown etiology characterized by non-caseating granulomata (1). Hutchison pioneered the dermatologic features in 1877; uveitis was first described in 1910 (2). Ocular diseases have been documented in up to $60 \%$ of patients with systemic sarcoidosis (3). Conversely, ocular disease can be the initial presentation in up to one third of patients (4). The commonest feature is uveitis which if untreated can result in permanent visual impairment. Definitive diagnosis can be challenging as there is no pathognomonic test, only constellations of clinical, laboratory, radiological features supported by histological evidence. The International Workshop on Sarcoidosis (IWOS) proposed guidelines to aid early diagnosis and timely intervention (5).

The authors present a 25-year-old male with bilateral panuveitis and, unusually, 'sarcoidal tattoo granuloma $(6,7)$. An early accurate diagnosis is important as it guides the therapeutic strategy to reduce morbidity and mortality. The report aims to highlight the 
ophthalmic features of sarcoidosis and the importance of a multidisciplinary team to improve both the diagnostics and therapeutics of this great masquerader.

\section{CASE REPORT}

A 25-year-old male of East Indian descent presented with a two weeks history of bilateral red eyes. The Snellen visual acuity was $6 / 4.8$ right eye and 6/7.5 left eye.

Slit lamp examination revealed cells and keratic precipitates in both anterior chambers; there were no synechia/ Koppe/Bussaca nodules; the intraocular pressures were $10 \mathrm{mmHg}$ bilaterally; the posterior segments were quiet. The assessment was acute anterior uveitis; topical prednisolone was commenced and a referral to the rheumatologist was recommended to investigate for an underlying systemic etiology. Preliminary screen was normal (Figure 1).

Three months later, while tapering the steroid drops, he reported: decreased vision and floaters bilaterally, raised and indurated tattoos (Figure 2a). The tattoos were done 8 years earlier. Ophthalmic examination revealed bilateral vitritis with snowballs in the left eye. He was referred to a dermatologist who performed a skin biopsy. The histopathologist reported histiocytic granulomatous infiltration (Figure 3). The ESR CRP and s-ACE were now elevated.

The clinical diagnosis of bilateral panuveitis together with granulomatous histopathology, elevated ACE level, negative TB quantiferon: suggested a diagnosis of definite ocular sarcoidosis, based on the criteria proposed by the International Workshop on Ocular Sarcoidosis (IWOS)in 2009 (Table 1). Systemic immunosuppression was initiated (prednisolone1mg/kg/day) along with recommended prophylaxis. While tapering the systemic steroids, prednisolone 30mg daily, he began experiencing floaters and decreased vision bilaterally. Mycophenolate mofetil $1 \mathrm{~g}$ 
bd was added as a steroid sparing agent. He has responded well on this regime with resolution of the clinical features (Figure 2b).

\section{DISCUSSION}

Norwegian dermatologist Caesar Boeck, in 1877, coined the term 'sarkoid' because of the cutaneous lesions' histological resemblance to a sarcoma $(7,8)$ It is a multi-systemic granulomatous inflammatory disorder of unknown etiology (1). Ninety percent (90\%) of patients have pulmonary involvement, cutaneous, ocular features or a combination of these findings $(8,9$, 10). It remains a diagnostic and therapeutic challenge as there is no single definitive investigation. The diagnosis relies on a constellation of clinical, laboratory, radiological features supported by histopathological evidence. Timely diagnosis is critical to reducing morbidity and mortality.

Although the inciting agent remains unknown, granulomas generally form to localise pathogens. The granulomas are discrete, compact collections of epithelioid cells rimmed by an outer zone of largely CD4+ cells (9). Studies confirm a genetic predisposition with evidence for the allelic variation at the $H L A-D R B 1$ locus as a major contributor (11).

The authors present a case of bilateral panuveitis with an unusual dermatologic manifestation of 'sarcoidal tattoo granuloma'. Sarcoid tattoo granulomas can present as plaques, nodules or papules. It was first documented by Maden in 1939. (12-15). Notably, $70 \%$ of these patients had systemic involvement at presentation. The theory as to whether the pigment is a trigger or target is, not surprisingly, equivocal. It is proposed that the pigment can either act as a 
nidus or due to low grade chronic inflammation, systemic sarcoidosis can develop in genetically predisposed individuals.

The presence of noncaseating granulomata is the gold standard for diagnosis (16). In the absence of histopathology, clinicians have the dilemma of diagnostic uncertainty. A systematic approach was proposed by the First International Workshop on Ocular Sarcoidosis (IWOS) in 2009 (5). There are four categories of diagnostic certainty: definite, presumed, probable and possible. In this case report, the clinical diagnosis of bilateral panuveitis together with granulomatous histopathology, elevated ACE level, negative TB quantiferon: suggested a diagnosis of definite ocular sarcoidosis.

Given the prominence of ophthalmic features, documented increased predisposition in Caribbean population and the threat of diagnostic uncertain, the authors aim to outline the ophthalmic features.

\section{Uveitis}

Uveitis is inflammation of the uveal tract which comprises the iris, ciliary body and choroid (17). Anatomical classification is as follows: anterior, intermediate, posterior and panuveitis.

\section{Anterior uveitis}

Anterior uveitis occurs in $22-70 \%$ of patients; it can be acute or chronic (18). The former presents with red eyes, photophobia and blurred vision, as did this patient. The later can be more dubious with very little symptoms until there are advanced eye diseases. In general, patients with acute anterior uveitis tend to be younger usually aged 20-35 years while chronic anterior sarcoid uveitis is found in those between $35-50$ years (19). 
The signs include anterior chamber cells, flare and/or large mutton fat keratic precipitates (Figure 4). The latter are small aggregates of inflammatory cells often seen in a gravitational distribution along the middle and inferior region of the corneal endothelium (Arlt's triangle) [20]. Worryingly, up $34 \%$ of patients with active uveitis can be asymptomatic (21). Chronicity is also associated with Busacca's nodules which are true granulomatous lesions found on the iris; Koeppe's nodules are limited to the pupillary margin (19).

The complications of uncontrolled uveitis are sight-threatening including secondary glaucoma, cataract or cystoid macula edema [Figure 5] (20). Cystoid macula edema can be caused by chronic uveitis and may be particularly refractory to conventional anti-inflammatory therapy (22).

Intermediate uveitis

Intermediate uveitis can be detected in $23-26 \%$ of patients with sarcoidosis. It involves inflammation occurring in the vitreous, pars plana and peripheral retina $(23,24)$. Patients with may be asymptomatic, complain of floaters and/or blurred vision. Pars planitis results in cellular accumulation in the vitreous known as "snowballs" and "string of pearls" (Figure 6) as demonstrated in this case.

\section{Posterior uveitis}

Posterior uveitis occurs in up to $28 \%$ of patients with ocular sarcoidosis $(25,26)$. Vitritis and periphlebitis ("candle wax drippings" or "taches de bouge") are the most common manifestations. Other features include secondary vascular occlusion and retinal granulomas. 


\section{Panuveitis}

Panuveitis involves inflammation of all areas with both the anterior and posterior segments. It occurs in 6-33\% of patients with sarcoidosis and is considered a poor prognostic factor (26-29). Diagnostic certainty is pivotal to planning an effective treatment strategy as seen in our patient.

\section{Neuro-ophthalmic disease}

Optic neuropathy is a rare sight-threatening complication of sarcoidosis. Patients complain of rapid decrease in vision. Disturbance of colour perception and contrast sensitivity may be detected (18). Optic nerve involvement can be associated with papillitis, papilledema and even granulomas of the optic head (19). Optic atrophy may be the ultimate result of optic nerve involvement hence aggressive treatment is recommended.

\section{Orbital and adnexal disease}

Eyelid or conjunctival granulomas are well reported in sarcoidosis [30]. Conjunctival biopsy may provide a simple and rapid tissue diagnosis with a positive yield of up to $40 \%$ especially with conjunctival follicles (31).

Lacrimal gland involvement can occur in $15-28 \%$ of patients with sarcoid eye disease (32). Enlargement of the affected lacrimal gland may be evident on palpation or rarely enlarge sufficiently to cause ptosis and/or proptosis (33). Biopsy of the lacrimal gland is also a very useful diagnostic tool.

\section{Treatment strategy}

Corticosteroids are the mainstay in the management of both systemic and/or ocular sarcoidosis. Topical corticosteroids are recommended for anterior uveitis. Systemic corticosteroids are 
initiated in the presence of posterior segment disease notably with systemic clinical features and/or bilateral uveitis.

In the presence of unilateral disease periocular steroids are an effective alternative in mild to moderate inflammation. More recently, intravitreal steroids have been demonstrated good efficacy. These modalities, however, do increase the risks of cataract and glaucoma.

Early introduction of a steroid sparing agent is considered good clinical practise as it reduces the long-term complication of oral steroids. Additionally, escalation of the immunosuppression treatment tier if the disease persists and/or becomes more aggressive must be done in a timely manner by the multi-disciplinary team. Other steroid sparing alternatives include cytotoxics (methotrexate, azathioprine, mycophenolate, leflunomide) and/or biologics such as (infliximab, adalimumab/rituximab) with the choice tailored as per the side-effect profile and specifics of the individual patient.

\section{CONCLUSION}

Sarcoidosis is a multisystemic granulomatous disease with both sight and life-threatening sequelae. Definitive diagnosis can be challenging as there is no pathognomonic test, only constellations of clinical, laboratory, radiological features supported by histological evidence. The International Workshop on Sarcoidosis proposed guidelines to aid early diagnosis and timely intervention.

Ocular sarcoidosis can be the presenting clinical feature in up to one third of patients; similarly for dermatological manifestation. Notably, $70 \%$ of patients with ocular sarcoidosis and/or sarcoid tattoo granuloma independently develop systemic sarcoidosis. Early diagnosis and 
timely intervention, inclusive of early introduction of a steroid sparing agent, reduces the morbidity and mortality. Equally important is the role of the multi-disciplinary team in elucidating the seemingly changing clinical scenario in these challenging patients. 


\section{REFERENCES}

1. Haimovic A, Sanchez M, Judson MA, Prystowsky S. Sarcoidosis: a comprehensive review and update for the dermatologist: part I. Cutaneous disease. J Am Acad Dermatol. 2012; 66(5):699.e1-18.

2. James DG, Sharma OP. From Hutchinson to now: a historical glimpse. Curr Opin Pulm Med. 2002; 8(5):416-23.

3. Lee SY, Lee HG, Kim DS, Kim JG, Chung H, Yoon YH. Ocular sarcoidosis in a Korean population. J Korean Med Sci. 2009; 24 (3):413-9.

4. Rothova A, Ocular involvement in sarcoidosis. The British Journal of Ophthalmology 2000; 84: 110-116.

5. Herbort CP, Rao NA, Mochizuki M. International criteria for the diagnosis of ocular sarcoidosis: results of the first International Workshop on Ocular Sarcoidosis (IWOS). Ocular immunology and inflammation 2009; 17: 160-169.

6. O'Beirne SL, O'Dwyer DN, Walsh SM, Dodd JD, Crotty TB, Donnelly SC. The lady with the dragon tattoo. Ir J Med Sci. 2016 Mar 9.

7. Sharma OP, Shigemitsu H. A historical sketch; life and time of Jonathan Hutchinson (1828-1913), the first sarcoidologist. Sarcoidosis Vasc Diffuse Lung Dis. 2008;25(2):715.

8. Iannuzzi MC, Rybicki BA, Teirstein AS. Sarcoidosis. N Engl J Med. 2007;357(21):215365.

9. Kumar V, Abbas AK, Fausto N, Mitchell RN (2007). Robbins Basic Pathology (8th ed.). Saunders.

10. Baughman RP, Lower EE, du Bois RM. Sarcoidosis. Lancet 2003; 361:1111. 
11. Rossman MD, Thompson B, Frederick M, et al. HLA-DRB1*1101: a significant risk factor for sarcoidosis in blacks and whites. Am J Hum Genet. 2003; 73(4):720-35.

12. Baumgartner M, Feldmann R, Breier F, Steiner A. Sarcoidal granulomas in a cosmetic tattoo in association with pulmonary sarcoidosis. J Dtsch Dermatol Ges. 2010 Nov; 8(11):900-2.

13. Papageorgiou PP, Hongcharu W, Chu AC. Systemic sarcoidosis presenting with multiple tattoo granulomas and an extra-tattoo cutaneous granuloma. J Eur Acad Dermatol Venereol. 1999 Jan; 12(1):51-3.

14. Weidman AI, Andrade R, Franks AG. Sarcoidosis. Report of a case of sarcoid lesions in a tattoo and subsequent discovery of pulmonary sarcoidosis. Arch Dermatol. 1966 Sep;94(3):320-5.

15. Hanada K, Chiyoya S, Katabira Y. Systemic sarcoidal reaction in tattoo. Clin Exp Dermatol 1985; 10(5):479-484.

16. Chan AS, Sharma OP, Rao NA, Review for disease of the year: immunopathogenesis of ocular sarcoidosis. Ocular immunology and inflammation 2010; 18: 143-151.

17. Mitchell DN, Wells A, Spiro SG et al. Sarcoidosis. CRC Press; 2012.

18. Bradley DA, Baughman RP, Raymond L, Kaufman AH. Ocular manifestations of sarcoidosis. Semin Respir Crit Care Med 2002; 23: 543-548.

19. Mayers M. Ocular sarcoidosis. Int Ophthalmol Clin 1990; 30: 257-263.

20. Silver MR, Messner LV. Sarcoidosis and its ocular manifestations. J Am Optom Assoc 1994; 65: 321-327. 
21. Pietinalho A, Ohmichi M, Lo"froos AB, Hiraga Y, Selroos O. The prognosis of pulmonary sarcoidosis in Finland and Hokkaido, Japan: a comparative five-year study of biopsyproven cases. Sarcoidosis Vasc Diffuse Lung Dis 2000; 17: 158-16.

22. Baughman RP, Teirstein AS, Judson MA, et al. Case Control Etiologic Study of Sarcoidosis (ACCESS) research group Clinical characteristics of patients in a case control study of sarcoidosis. Am J Respir Crit Care Med 2001; 164 (10 Pt 1): 1885-1889.

23. Landers PH. Vitreous lesions in Boeck's sarcoid. Am J Ophthalmol. 1949; 32:1740-1.

24. Boyd SR, Young S, Lightman S. Immunopathology of the noninfectious posterior and intermediate uveitides. Surv Ophthalmol 2001; 46: 209-233.

25. Drent M, Costabel U. European Respiratory Monograph 32: Sarcoidosis. European Respiratory Society; 2005.

26. Obenauf CD, Shaw HE, Sydnor CF, Klintworth GK. Sarcoidosis and its opthalmic manifestations. Am J Opthalmol 1978; 86: 648-655.

27. Bansal R, Gupta V, Gupta A. Current approach in the diagnosis and management of panuveitis. Indian J Ophthalmol. 2010; 58(1):45-54.

28. Jabs DA, Johns CA. Ocular involvement in chronic sarcoidosis. Am J Opthalmol 1986; 102: $297-301$.

29. Dana MR, Merayo-Lloves J, Schaumberg DA, Foster CS. Prognosticators for visual outcome in sarcoid uveitis. Ophthalmology. 1996; 103:1846-53.

30. Hall JG, Cohen KL (1995) Sarcoidosis of the eyelid skin. Am J Ophthalmol119:100-101.

31. Spaide RF, Ward DL. Conjunctival biopsy in the diagnosis of sarcoidosis. Br $\mathbf{J}$ Ophthalmol. 1990;74(8):469-71. 
32. Prabhakaran VC, Saeed P, Esmaeli B, et al. Orbital and adnexal sarcoidosis. Arch Ophthalmol 2007;125: 1657-1662.

33. Sacher M, Lanzieri CF, Sobel L (1984) Computed tomograhy of bilateral lacrimal gland sarcoidosis. J Comp Ass Tomograph 8:213-215.

Table 1: Systematic approach proposed by the First International Workshop on Ocular Sarcoidosis (IWOS) in 2009 to improve diagnostic certainty

\section{A: Clinical signs suggestive of ocular sarcoidosis}

I. Mutton fat keratic precipitates (KPs) and/or iris nodules (Koeppa/ Busacca) at the pupillary margin or in stroma

II. Trabecular meshwork nodules and/or tent-shaped peripheral anterior synechiae

III. Snowballs/ string of pearl vitreous opacities

IV. Multiple chorioretinal peripheral lesions (active or atrophic)

V. Nodular and/or segmental periphlebitis and/or macroaneurysm

VI. Optic disc nodule/ granuloma and/or solitary choroidal nodule

VII. Bilaterally on clinical examination or on investigation

\section{B: Laboratory investigations in suspected ocular sarcoidosis}

1. Negative tuberculin test in BCG vaccinated patient

2. Elevated Serum angiotensin converting enzyme (ACE) and/or elevated serum lysozyme

3. Chest X-ray- presence of bilateral hilar lymphadenopathy (BHL)

4. Abnormal liver enzymes: any two

5. Chest $\mathrm{CT}$ scan in patients with negative chest X-ray

\section{C: Diagnostic criteria for ocular sarcoidosis}

Definite: Biopsy supported diagnosis with a compatible uveitis Presumed: Biopsy not done, presence of BHL with a compatible uveitis Probable: Biopsy not done, BHL negative, presence of three clinical signs and two positive investigational tests. Possible: Biopsy negative, four of the suggestive intraocular signs and two if the investigations are positive. 


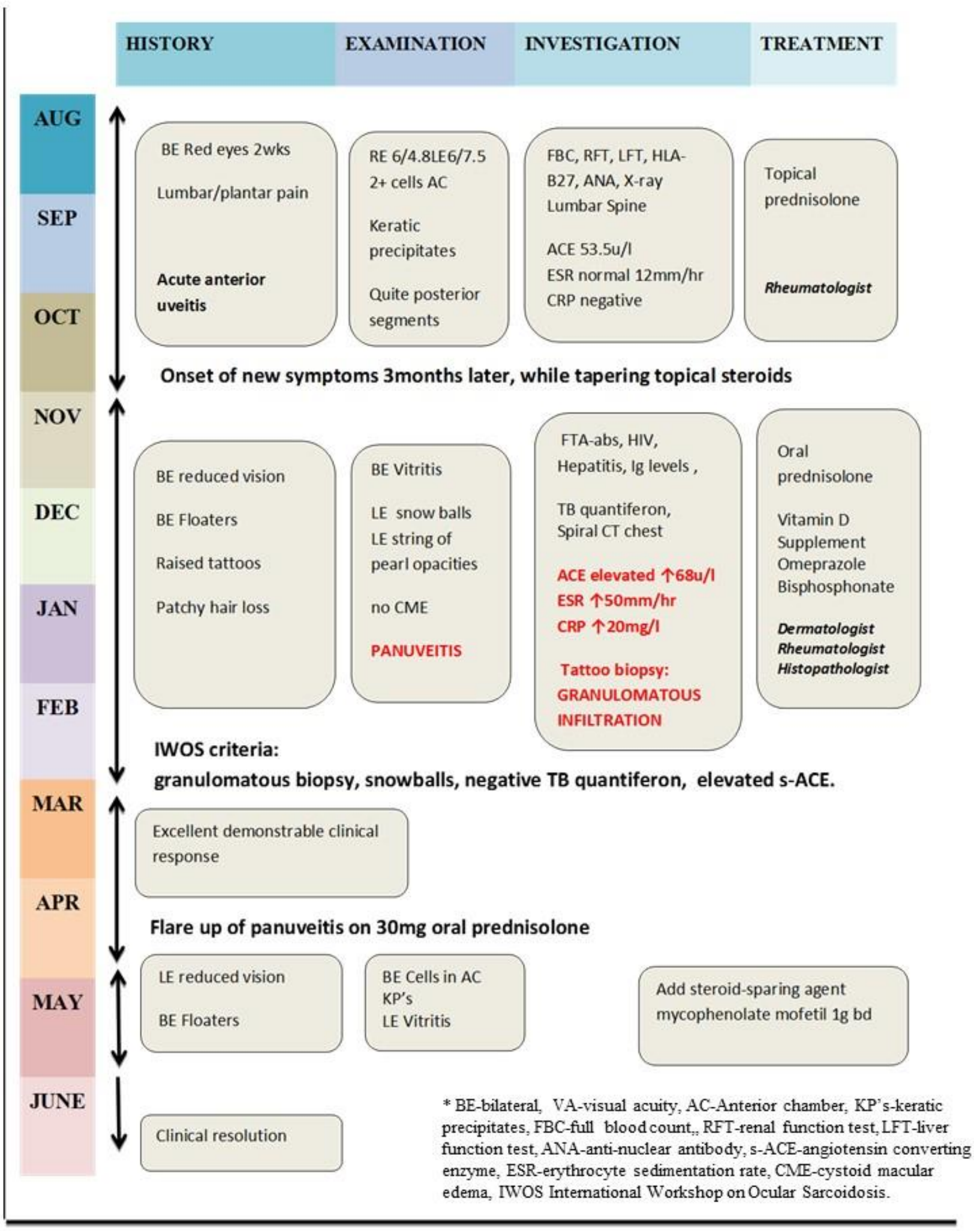

Fig. 1: Pictograph of clinical course. 


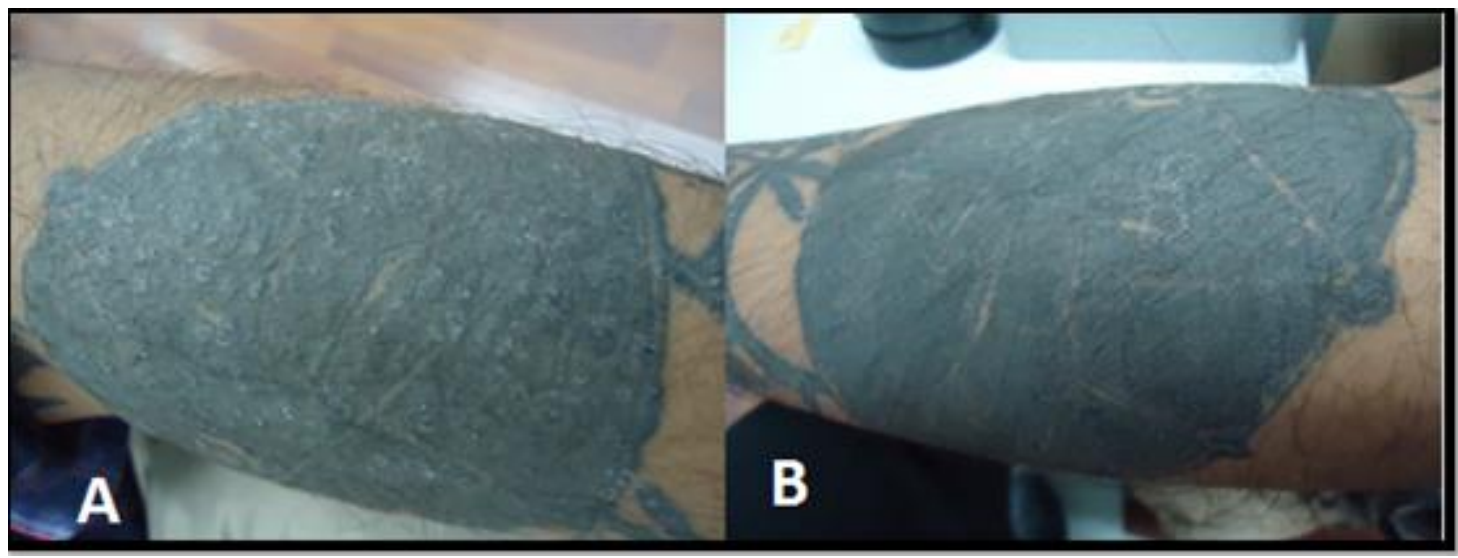

Fig. 2: Sarcoid tattoo granuloma (A) indurated tattoo before systemic immunosuppression, (B) clinical response on oral prednisolone three months later.

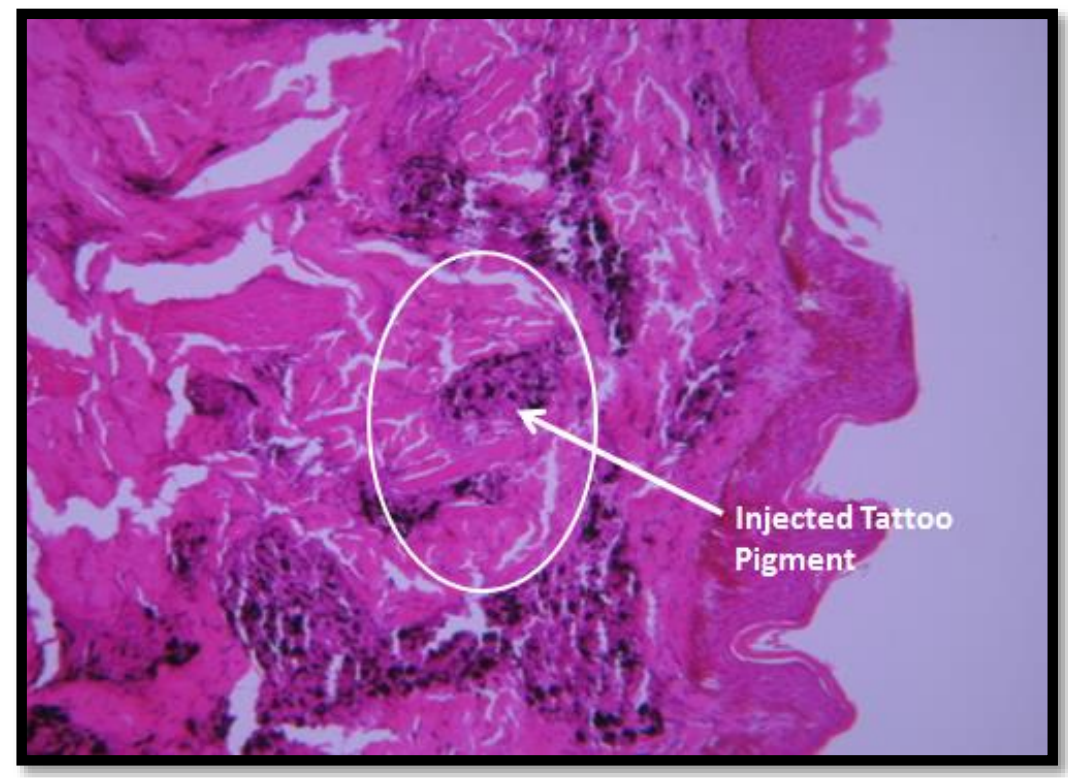

Fig. 3: Hematoxylin and eosin slide demonstrating histiocytic granulomatous infiltration around pigment in forearm biopsy on this patient. 


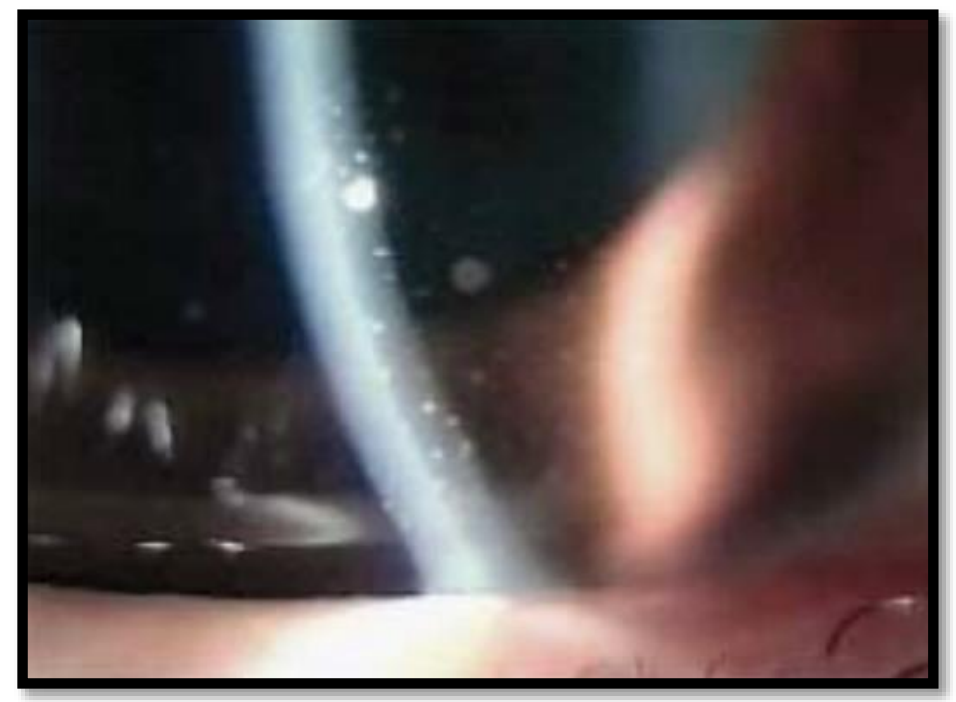

Fig 4: An example of keratic precipitates on slit examination [22] (not the patient discussed in this case report)

Reproduced with permission of the European Respiratory Society @: European Respiratory Society Monograph, Sarcoidosis, Edited by M. Drent and U. Costabel. Book | Published in 2005, DOI: 10.1183/1025448x.erm3205, 12. Clinical aspects of ocular sarcoidosis, K. Ohara, M.A. Judson and R.P. Baughman, 10.1183/1025448x.00032012

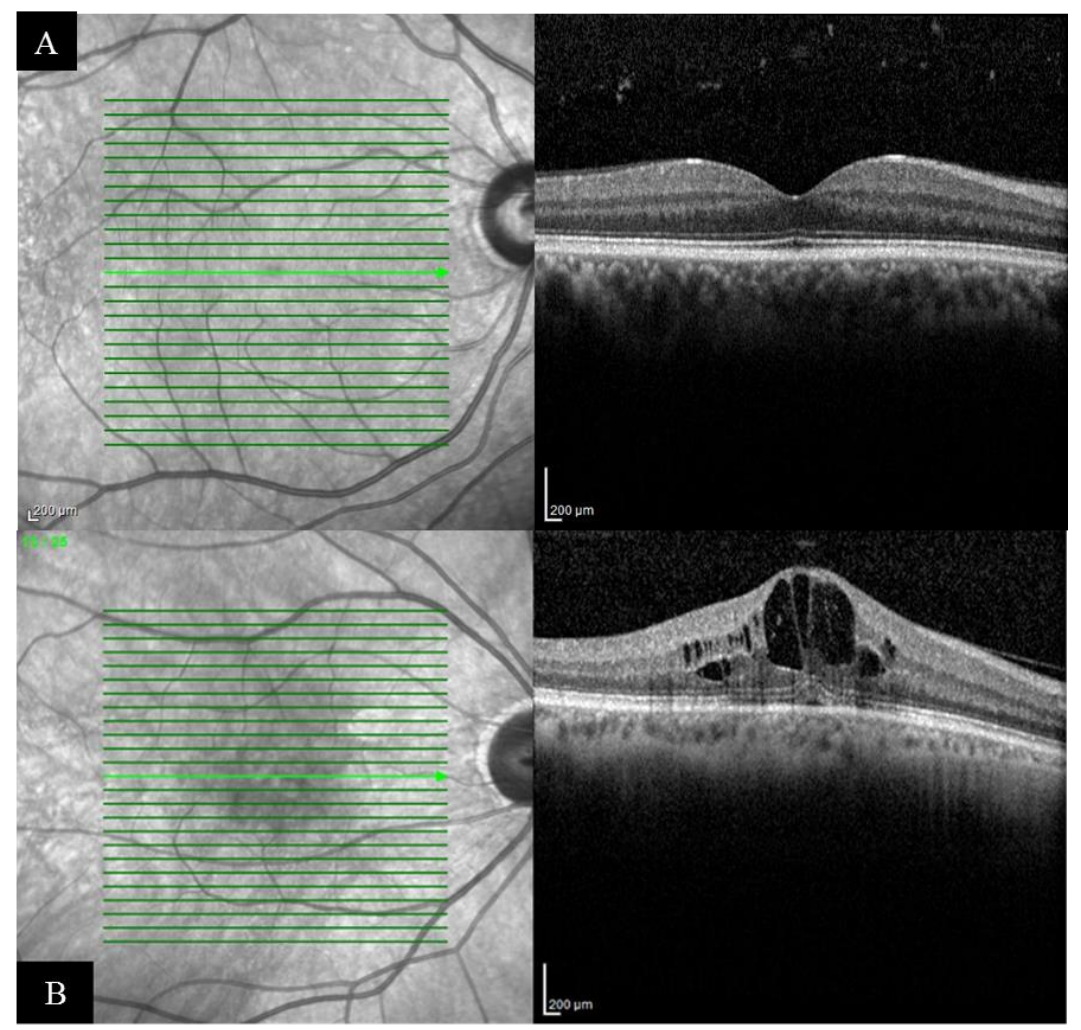

Fig 5: (A) Normal Optical Coherence Tomography (OCT) macular scan, (B) OCT cross section of the macular demonstrating cystoid macular edema a sight-threatening complication of sarcoid uveitis. 


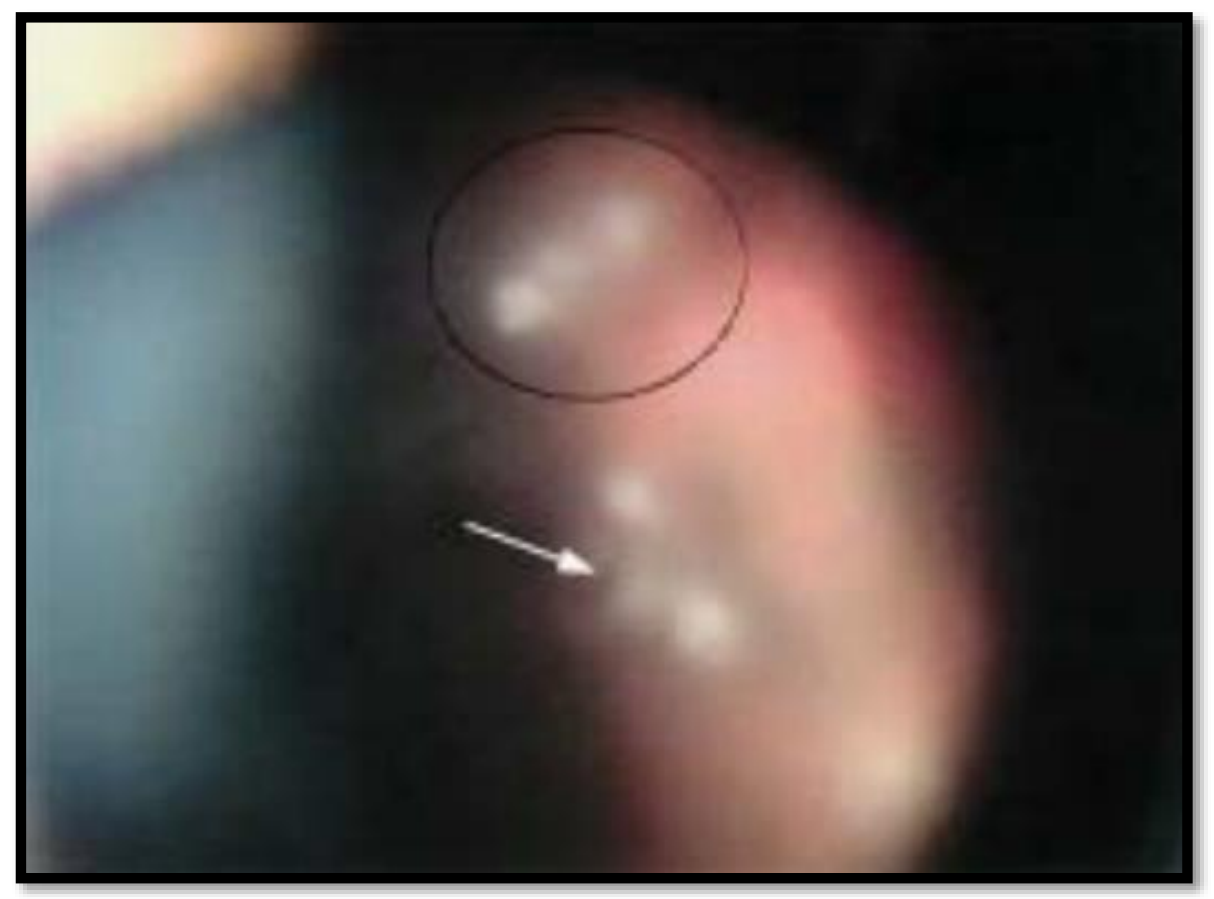

Fig. 6: An example of snowball (arrow) and a string of pearls-like vitreous opacities (circled) (not patient discussed in this case report) $[22,36]$

Reproduced with permission of the European Respiratory Society @: European Respiratory Society Monograph, Sarcoidosis, Edited by M. Drent and U. Costabel. Book | Published in 2005, DOI: 10.1183/1025448x.erm3205, 12. Clinical aspects of ocular sarcoidosis, K. Ohara, M.A. Judson and R.P. Baughman, 10.1183/1025448x.00032012 